\title{
Front Matter: 11259
}

, "Front Matter: 11259," Proc. SPIE 11259, Solid State Lasers XXIX:

Technology and Devices, 1125901 (2 April 2020); doi: 10.1117/12.2569742

SPIE. Event: SPIE LASE, 2020, San Francisco, California, United States 


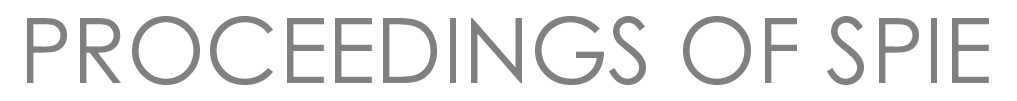

\section{Solid State Lasers XXIX: Technology and Devices}

W. Andrew Clarkson

Ramesh K. Shori

Editors

4-6 February 2020

San Francisco, California, United States

Sponsored and Published by

SPIE 
The papers in this volume were part of the technical conference cited on the cover and title page. Papers were selected and subject to review by the editors and conference program committee. Some conference presentations may not be available for publication. Additional papers and presentation recordings may be available online in the SPIE Digital Library at SPIEDigitalLibrary.org.

The papers reflect the work and thoughts of the authors and are published herein as submitted. The publisher is not responsible for the validity of the information or for any outcomes resulting from reliance thereon.

Please use the following format to cite material from these proceedings:

Author(s), "Title of Paper," in Solid State Lasers XXIX: Technology and Devices, edited by W. Andrew Clarkson, Ramesh K. Shori, Proceedings of SPIE Vol. 11259 (SPIE, Bellingham, WA, 2020) Seven-digit Article CID Number.

ISSN: 0277-786X

ISSN: 1996-756X (electronic)

ISBN: 9781510632813

ISBN: 9781510632820 (electronic)

Published by

SPIE

P.O. Box 10, Bellingham, Washington 98227-0010 USA

Telephone +1 3606763290 (Pacific Time) · Fax +1 3606471445

SPIE.org

Copyright (c) 2020, Society of Photo-Optical Instrumentation Engineers.

Copying of material in this book for internal or personal use, or for the internal or personal use of specific clients, beyond the fair use provisions granted by the U.S. Copyright Law is authorized by SPIE subject to payment of copying fees. The Transactional Reporting Service base fee for this volume is $\$ 21.00$ per article (or portion thereof), which should be paid directly to the Copyright Clearance Center (CCC), 222 Rosewood Drive, Danvers, MA 01923. Payment may also be made electronically through CCC Online at copyright.com. Other copying for republication, resale, advertising or promotion, or any form of systematic or multiple reproduction of any material in this book is prohibited except with permission in writing from the publisher. The CCC fee code is 0277$786 \times / 20 / \$ 21.00$.

Printed in the United States of America by Curran Associates, Inc., under license from SPIE.

Publication of record for individual papers is online in the SPIE Digital Library.

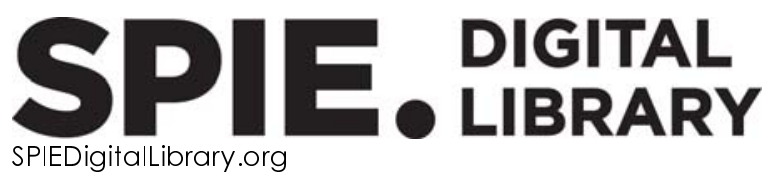

Paper Numbering: Proceedings of SPIE follow an e-First publication model. A unique citation identifier (CID) number is assigned to each article at the time of publication. Utilization of CIDs allows articles to be fully citable as soon as they are published online, and connects the same identifier to all online and print versions of the publication. SPIE uses a seven-digit CID article numbering system structured as follows:

- The first five digits correspond to the SPIE volume number.

- The last two digits indicate publication order within the volume using a Base 36 numbering system employing both numerals and letters. These two-number sets start with 00, 01, 02, 03, 04, 05, 06, 07, 08, 09, OA, OB ... 0Z, followed by 10-1Z, 20-2Z, etc. The CID Number appears on each page of the manuscript. 


\section{Contents}

$\begin{array}{ll}\text { vii } & \text { Authors } \\ \text { ix } & \text { Conference Committee }\end{array}$

EYE SAFE AND MID-IR LASERS I

$1125902 \quad 1.34 \mu \mathrm{m} \mathrm{Nd:YVO} 4$ laser passively Q-switched by V:YAG and optimized for lidar [1 1259-1]

$1125905 \quad 1.7 \mu \mathrm{m}$ diode-pumped Tm:GGAG and Tm, Ho:GGAG 2-2.1 $\mu \mathrm{m}$ laser [1 1259-4]

1125906 Passively Q-switched $10 \mathrm{~mJ}$ Tm:YLF laser with efficient OPO conversion to mid-IR [1 1259-5]

1125907 Compact $12 \mathrm{~mJ}$ mid-IR pulsed source using an intracavity KTA OPO followed by a CSP OPA [11259-6]

EYE SAFE AND MID-IR LASERS II

11259 OC Crystal host engineering for transition metal lasers [1 1259-11]

STRUCTURED BEAMS

11259 OE Q-switched vortex laser using a Sagnac interferometer as an output coupler [11259-13]

11259 OG Diode-pumped Yb:CALGO laser with conical refraction output [1 1259-15]

$11259 \mathrm{OH} \quad$ High purity twisted light from a metasurface solid state resonator [1 1259-16]

NOVEL LASER CONCEPTS

11259 0J Narrow linewidth tunable and dual wavelength compact Alexandrite laser [1 1259-18]

11259 OK Nd:YLF/KGW intracavity Raman laser in DBMC configuration emitting at 1147 and $1163 \mathrm{~nm}$ in TEMoo [1 1259-19] 
PULSED LASERS I

$11259 \mathrm{ON}$ Scalable amplification with a high gain $\mathrm{x}$ energy product at room temperature using a thick slab of $\mathrm{Yb}^{3+}:$ YAG [1 $\left.1259-22\right]$

11259 OP Passively Q-switched Nd:YVO4 laser operating at 914 nm [11259-24]

$112590 Q \quad$ Ultra-compact >100kHz Q-switched Alexandrite lasers [1 $1259-25]$

PULSED LASERS II

11259 OT Fiber-coupled high-power diode-pumped solid-state lasers for laser cleaning [1 1259-28]

11259 OU A fiber/solid-state hybrid laser system for ion beam control in a particle accelerator [1 1259-29]

11259 OW High average power passively Q-switched Yb:YAG micro-laser [1 1259-31]

11259 OY Active pulse underwater vision system [11259-33]

\section{LASER MATERIAL CHARACTERIZATION I}

$112590 Z \quad Y b$ concentration influence of on thermal lensing in Yb:LUAG and Yb:YAG lasers at cryogenic temperatures: modeling and experimental study [1 1259-34]

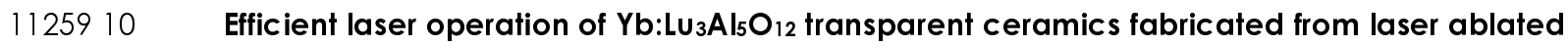
nanopowders [11259-35]

1125911 Growth, spectroscopy and laser operation of $\mathrm{Yb}^{3+}, \mathrm{Na}^{+} / \mathrm{Li}^{+}$-codoped CNGG-type garnets promising for ultrafast lasers [1 1259-36]

\section{LASER MATERIAL CHARACTERIZATION II}

1125913 Recent progress in mechanically Q-switched $2.94 \mu \mathrm{m}$ Er:YAG - promising pump source for

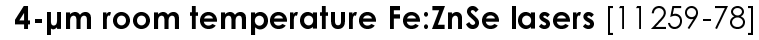

1125915 Time-gated measurements of fusion-class laser beam profiles [11259-39]

1125917 Spatially resolved B-integral measurements on the NIF laser [1 1259-41]

1125918 Precision diagnostic system enhancements and recommissioning for advanced laser beam characterization at the National Ignition Facility [1 1259-42] 
1125919 Tunable $\mathrm{Cr}^{2+}, \mathrm{Fe}^{2+}: \mathrm{Zn}_{1-x} \mathrm{Mn}_{\mathrm{x}} \mathrm{Se}(\mathrm{x}=\mathbf{0 . 0 5})$ and $(\mathrm{x}=0.3)$ lasers around $4.4 \mu \mathrm{m}$ at $78 \mathrm{~K}$ pumped by a $1.94 \mu \mathrm{m}$ Tm:fiber laser via $\mathrm{Cr}^{2+} \rightarrow \mathrm{Fe}^{2+}$ energy transfer [1 1259-43]

$112591 \mathrm{~A} \quad$ Spectroscopic characterization of Fe: $\mathrm{ZnAl}_{2} \mathrm{O}_{4}, \mathrm{Fe}: \mathrm{MgAl}_{2} \mathrm{O}_{4}$ and Fe:InP crystals for mid-IR laser applications [1 1259-44]

\section{ULTRAFAST LASERS I}

$112591 \mathrm{~F} \quad$ Femtosecond $100 \mathrm{~W}$-level OPCPAs from near-IR to short-wave-IR wavelengths [11259-49]

ULTRAFAST LASERS II

$112591 \mathrm{H} \quad$ Operation of a novel, dual function thin slab ultrafast amplifier at $1030 \mathrm{~nm}, 515 \mathrm{~nm}$ and $343 \mathrm{~nm}$ [11259-51]

$1125911 \quad 0.5$ terawatt laser based on a hybrid architecture for high energy diode-pumped lasers delivering sub-500 fs pulses [1 1259-52]

$112591 \mathrm{~J} \quad 10$ petawatt lasers for extreme light applications [11259-53]

$112591 \mathrm{~K} \quad$ High temporal contrast, diode pumped, femtosecond laser providing 200fs, 1053nm pulses for seeding large scale Nd:glass laser systems [1 1259-54]

$112591 \mathrm{~L} \quad$ High power CEP-stable OPCPA at 800nm [11259-55]

UV-VIS LASERS

$112591 \mathrm{M} \quad \mathbf{2} \mathbf{~ k W ~ c w}$ laser in the green wavelength regime for copper welding (Invited Paper) [1 1259-56]

$1125910 \quad$ Multiple and selectable wavelength green laser generation based on coaxial diode-endpumping [11259-58]

11259 1P High efficiency gallium nitride laser diode pumped CW ruby laser [1 1259-59]

POSTER SESSION

$112591 \mathrm{Q}$ Temperature influence on Er:GGAG crystal spectroscopic properties and its lasing at $3 \mu \mathrm{m}$ [1 1259-60]

11259 1R Efficient composite Nd:YVO/Nd:GVO laser with in-band pumping [1 1259-61]

11259 1S Dual-wavelength Yb:CALGO laser with wavelength spacing tunability [1 1259-62] 
$112591 \mathrm{~T} \quad$ Performance of diode-pumped Yb:YAP lasers with different crystal orientations [11259-63]

$112591 \mathrm{U} \quad$ Generation of THz frequency offset with dual-wavelength Yb:KGW laser [11259-64]

$112591 \mathrm{~V} \quad$ Simplified cavity design for KLM Ti:sapphire oscillators [11259-65]

$112591 \mathrm{~W}$ Thermal lensing in diode-pumped Yb:CALGO and Yb:KGW lasers [1 1259-66]

$112591 \mathrm{X} \quad$ Programmable, pulse shaped diode laser [11259-67]

1125921 Samarium-doping concentration influence on spectroscopic parameters of Sm:YAG crystal [1 1259-71]

$1125923 \quad$ Fe:ZnMnTe laser generating around 5 um at 78 K [11259-73]

1125925 Generation of $\mathbf{4 0} \mathbf{W}, \mathbf{4 0 0}$ fs pulses at $1 \mathrm{MHz}$ repetition rate from efficient, room temperature Yb:YAG double-pass amplifier seeded by fiber CPA system [11259-75]

1125927 Multi-watt continuous-wave and passively Q-switched Tm:CaYAlO4 micro-lasers [1 $1259-77]$

$1125928 \quad$ Laser spectroscopic and saturation properties of GR1 centers in synthetic diamond [1 1259-79]

1125929 73-fs SESAM mode-locked Tm,Ho:CNGG laser at $2061 \mathrm{~nm}$ [11259-80]

11259 2D Spectral density contrast in DPSS and ECD lasers for quantum and other narrow-linewidth applications [1 1259-84] 


\section{Authors}

Numbers in the index correspond to the last two digits of the seven-digit citation identifier (CID) article numbering system used in Proceedings of SPIE. The first five digits reflect the volume number. Base 36 numbering is employed for the last two digits and indicates the order of articles within the volume. Numbers start with 00, 01, 02, 03, 04, 05, 06, 07, 08, 09, 0A, 0B...0Z, followed by 10-1Z, 20-2Z, etc.

Aguiló, Magdalena, 10, 11, 27

Akbari, R., 0G, 1S, 1T, 1U, 1V, 1W

Ambrosio, Antonio, $\mathrm{OH}$

Bae, Ji Eun, 27

Balabanov, Stanislav, $1 \mathrm{~A}$

Baleanu, A., $1 \mathrm{~J}$

Banici, R., $1 \mathrm{~J}$

Bartulevicius, T., 25

Basyrova, Liza, 10

Baumann, Frank, 1M

Belyaev, Alexander, 1A

Berry, Patrick A., 0C

Birch, Rolf B., $1 \mathrm{H}$

Birkin, David, $1 \mathrm{H}$

Bisch, Stefanie, 1M

Boháček, Pavel, 05, 1Q

Boudjemaa, L., $1 \mathrm{~J}$

Boullet, Johan, ON

Brisset, Jean-Gabriel, 11

Brockmann, Rüdiger, $1 \mathrm{M}$

BUß, J. H., IF, $1 \mathrm{~L}$

Cai, Huaqiang, 11

Caldararu, C., $1 \mathrm{~J}$

Camy, Patrice, 11

Capasso, Federico, $\mathrm{OH}$

Casagrande, $0 ., 1 \mathrm{~J}$

Čech, Miroslav, 19

Chalus, O., $1 \mathrm{~J}$

Chen, Mengting, 27

Chen, Weidong, 29

Chen, Zhenqiang, 27

Chinn, Stephen R., OW

Cohen, S. J., 15, 17, 18

Cole, Brian, 06, 07

Cook, Gary, 0C

Courjaud, Antoine, 11

Coyne, Bryce K., 1 A

Dai, Shibo, 27

Dai, Xiaojun, 11

Damzen, Michael J., OE, OJ, OQ

Dancus, I., $1 \mathrm{~J}$

De Boisdeffre, B., $1 \mathrm{~J}$

Dekorsy, Thomas, OP

Derycke, C., $1 \mathrm{~J}$

Di Nicola, J. M., 15, 17, 18

Díaz, Francesc, 10, 11, 27

Dold, Eva-Maria, 1M

Doroshenko, Maxim E., 19, 23

Durand, Magali, 11
Erdmann, Rainer, $1 \mathrm{X}$

Erickson, M. A., 15, 17, 18

Evans, Jonathan W., OC

Fedorov, Vladimir, 13, 1A, 28

Fedorova, K. A., OG

Ferreira, Merilyn S., OK

Fey, Paul, $1 \mathrm{X}$

Fibrich, Martin, 05

Folta, J. A., 15, 17, 18

Forbes, Andrew, $\mathrm{OH}$

Fulford, Benjamin, $1 \mathrm{H}$

Gavory, Bastien, 11

Geberbaver, Jan Willem T., OE

Ghenuche, P., $1 \mathrm{~J}$

Ginis, Vincent, $\mathrm{OH}$

Goldberg, Lew, 06, 07, 0W

Golz, T., 1F, 1L

Gontier, Emilien, 11

Gottesman, N. S., 18

Gradinariu, A., $1 \mathrm{~J}$

Grguraš, I., 1F, $1 \mathrm{~L}$

Griebner, Uwe, 10, 11, 27, 29

Gruzevich, Y. K., OY

Guina, Mircea, 29

Handler, A. D., 15, 17, 18

Härkönen, Antti, 29

Harris, Thomas R., OC

Havlák, Lubomír, 05, 1 Q

Hays, Alan, 06, OW

Huang, Yao-Wei, $\mathrm{OH}$

Hurd, E. R., 15

Jambunathan, Venkatesan, 27

Jelínek, Michal, 19, 23

Jelínková, Helena, 05, 0Z, 19, 1Q, 21, 23

Jolly, Alain, ON

Jurek, Karel, 05, 1Q

Kaiser, Elke, $1 \mathrm{M}$

Kane, Thomas J., 02

Kapitch, Nick, 21

Karki, Krishna, 13, 1A

Karpushko, F., 2D

Kerridge-Johns, William R., OE

Khodakovskaia, M. S., OY

Khodakovskii, V. N., OY

Killi, Alexander, 1M

King, Vernon, 06

Kolliopoulos, G., $1 \mathrm{~J}$

Kopf, Daniel, $1 \mathrm{~K}$

Kovalenko, Nazar O., 19, 23 
Kratochvíl, Jan, 05, 1Q

Krupke, William F., IP

Kubeček, Václav, 19

Kulcsar, Gabor, $1 \mathrm{~K}$

Lanier, T. E., 15, 17, 18

Lauritsen, Kristian, $1 \mathrm{X}$

Laux, S., $1 \mathrm{~J}$

Leach, Jeffrey, OW

Lee, Jason R., $1 \mathrm{H}$

Liu, Kefei, 10

Liu, Yang, 10

Liu, Yun, OU

Loiko, Pavel, 10, 11, 1T, 1W, 27, 29

Lucianetti, Antonio, 27

Lureau, F., $1 \mathrm{~J}$

Machinet, Guillaume, ON

Madeikis, K., 25

Major, A., 0G, 1R, 1S, 1T, 1U, 1V, 1W, 27

Maksimov, Roman, 10

Markham, Matthew, 28

Martyshkin, Dmitry, 13

Mateos, Xavier, 10, 11, 27, 29

Matras, G., $1 \mathrm{~J}$

McCarthy, John C., 07

McDaniel, Sean A., OC

McIntosh, Chris, OW

Michailovas, A., 25

Mirov, Sergey, 13, 1A, 28

Mocek, Tomas, 27

Morbieu, T., $1 \mathrm{~J}$

Nadimi, M., IR

Naegele, Marco, OP

Naidoo, Darryl, $\mathrm{OH}$

Naziru, A., $1 \mathrm{~J}$

Neagu, L., $1 \mathrm{~J}$

Nejezchleb, Karel, 0Z, 21

Němec, Michal, 05, 19, 1Q, 21

Nettleton, John, 07

Nikl, Martin, 05, 1Q

Olejniczak, B. L., 15, 17

Onyenekwu, C., IR

Ordoñez, M. E., 18

Pan, Zhongben, 11, 29

Park, Tae Gwan, 27

Pask, Helen M., OK

Pellegrina, A., $1 \mathrm{~J}$

Petrov, Valentin, 10, 11, 27, 29

Pomeranz, Leonard A., 07

Prandolini, M. J., 1F, 1L

Pricking, Sebastian, 1M

Qiao, Hongzhan, 10

Qiu, Cheng-Wei, $\mathrm{OH}$

Radier, C., $1 \mathrm{~J}$

Rafailov, E. U., OG

Rakhman, Abdurahim, OU

Ren, Yuan, OT

Rey, G., 1J

Reza, Md. A. R., 0G, is

Ricaud, S., $1 \mathrm{~J}$

Ridderbusch, Heiko, OP
Riedel, Robert, 1F, $1 \mathrm{~L}$

Říha, Adam, 19, 23

Rotermund, Fabian, 27

Saci, Abdelhak, 11

Schepler, Kenneth L., OC

Schönau, Thomas, $1 \mathrm{X}$

Schranz, Erdal, 1K

Schulz, M., 1F, 1L

Schunemann, Peter G., 07

Sephton, Bereneice, $\mathrm{OH}$

Serres, Josep Maria, 10, 11, 27

Sevillano, Pierre, 11

Sheng, Quan, 10

Shitov, Vladislav, 10

Simon-Boisson, C., $1 \mathrm{~J}$

Smolski, Viktor, 1A

Sokolovskii, G. S., OG

Sroor, Hend, $\mathrm{OH}$

Stites, Ronald W., OC

Stoppel, Klaus, OP

Subedi, Shova, 13, 1A, 28

Šulc, Jan, 05, 0Z, 1Q, 21, 23

Suomalainen, Soile, 29

Švejkar, Richard, IQ

Szutor, B., 2D

Tate, T. H., 15

Tawy, Goronwy, 0J, 0Q

Terzin, Igor S., 19, 23

Trunda, Bohumil, 05, 1Q

Ursescu, D., $1 \mathrm{~J}$

Vallés, Adam, $\mathrm{OH}$

Veselis, L., 25

Veselský, Karel, $0 Z$

Vyhlídal, David, 23

Wang, Li, 29

Wang, Yicheng, 11, 29

Wang, Yong, OT

Wegner, P. J., 15, 17, 18

Wetter, Niklaus U., OK

Widmayer, C., 15, 17

Williams, W., 17

$\mathrm{Xu}$, Degang, 10

$X \cup, J ., 1 T$

$X \cup$, Jun, 27

XU, Xiaodong, 1T, 27

Yang, Dewang, OT

Yang, S. T., 15, 17, 18

Yao, Jianquan, 10

Yuan, Hualei, 11

Yue, Fangxin, 27

Zaske, Sebastian, $1 \mathrm{M}$

Zavadilová, Alena, 21

Zawilski, Kevin T., 07

Zhan, Qiwen, $\mathrm{OH}$

Zhao, Yongguang, 29

Zhong, Kai, 10

Zweiback, Jason, $1 \mathrm{P}$ 


\section{Conference Committee}

Symposium Chairs

Beat Neuenschwander, Berner Fachhochschule Technik und Informatik (Switzerland)

Xianfan Xu, Purdue University (United States)

Craig B. Arnold, Princeton University (United States)

Takunori Taira, Institute for Molecular Science (Japan)

Symposium Co-chairs

Craig B. Arnold, Princeton University (United States)

Takunori Taira, Institute for Molecular Science (Japan)

Program Track Chairs

Kunihiko Washio, Paradigm Laser Research Ltd. (Japan)

John Ballato, Clemson University (United States)

Conference Chairs

W. Andrew Clarkson, University of Southampton (United Kingdom)

Ramesh K. Shori, SPAWAR Systems Center (United States)

Conference Program Committee

Gary Cook, Air Force Research Laboratory (United States)

Dennis G. Harris, Dennis Harris Associates (United States)

Helena Jelínková, Czech Technical University in Prague (Czech Republic)

Christian Kränkel, Leibniz-Institut für Kristallzüchtung (Germany)

Jacob I. Mackenzie, University of Southampton (United Kingdom)

Markus Pollnau, University of Surrey (United Kingdom)

Narasimha S. Prasad, NASA Langley Research Center (United States)

Bojan Resan, Fachhochschule NordWestschweiz (Switzerland)

Nikolay E. Ter-Gabrielyan, U.S. Army Research Laboratory

(United States)

Session Chairs

1 Eye Safe and Mid-IR Lasers I

Ramesh K. Shori, Naval Information Warfare Center Pacific

(United States) 
2 Eye Safe and Mid-IR Lasers II

Ramesh K. Shori, Naval Information Warfare Center Pacific (United States)

3 Structured Beams

Dennis G. Harris, Dennis Harris Associates (United States)

4 Novel Laser Concepts

Narasimha S. Prasad, NASA Langley Research Center (United States)

5 Pulsed Lasers I

W. Andrew Clarkson, Optoelectronics Research Center (United Kingdom)

6 Pulsed Lasers $\|$

W. Andrew Clarkson, Optoelectronics Research Center (United Kingdom)

7 Laser Material Characterization I

Nikolay E. Ter-Gabrielyan, U.S. Army Combat Capabilities Development Command (United States)

8 Laser Material Characterization II

Nikolay E. Ter-Gabrielyan, U.S. Army Combat Capabilities Development Command (United States)

9 Ultrafast Lasers I

W. Andrew Clarkson, Optoelectronics Research Center (United Kingdom)

10 Ultrafast Lasers $\|$

Helena Jelínková, Czech Technical University in Prague (Czech Republic)

11 UV-VIS Lasers

Helena Jelínková, Czech Technical University in Prague (Czech Republic) 